\title{
Open Access und die Re-Kontextualisierung des Bibliothekserwerbungsetats
}

\author{
Dr. Ralf Schimmer, Max Planck Digital Library
}

\begin{abstract}
In diesem Beitrag wird der aktuelle Stand der Open Access-Debatte aufgegriffen und die Frage gestellt, welche strategisch-organisatorischen Anforderungen insbesondere hinter den zunehmenden Forderungen nach einer Transformation des traditionellen Subskrip1tionsmodells hin zu einem Publikationskostenansatz stehen. Ohne proaktives Handeln und bewusste Vorkehrungen seitens der Wissenschaftsorganisationen ist ein solcher Wandel kaum herbeizuführen. Gleichermaßen wichtig ist die Erkenntnis, dass sich die notwendigen Maßnahmen nicht in der Einrichtung von Publikationsfonds erschöpfen, sondern auch die ökonomischen Beziehungen zu den Verlagen neu zu organisieren sind. Eine entscheidende Rolle kommt hier dem Erwerbungsetat der Bibliotheken zu. Es gilt, dieses für die Transformation entscheidende Finanzreservoir in den sich abzeichnenden Open Access-

Organisationszusammenhängen neu zu kontextualisieren.

Schlüsselwörter: Open Access; Goldener Weg; Publikationskosten; Erwerbungsetat; Bibliothekspolitik
\end{abstract}

\section{Die strategisch-organisatorischen Implikationen hinter den Open Access- Entwicklungen}

Alle Anzeichen deuten darauf hin, dass sich die Open Access-Debatte immer mehr auf den goldenen Weg des genuinen Open Access-Publizierens zuspitzt.1 Es häufen sich die Rufe nach einer generellen Überwindung des traditionellen Subskriptionsmodells zugunsten eines Geschäftsmodells, bei dem die Kosten nicht mehr über den lesenden Zugriff, sondern über eine Finanzierung der Publikationen getragen werden. Auch an vielen deutschen Wissenschaftseinrichtungen werden entsprechende Publikationsfonds eingerichtet. Es ist jedoch bereits in der Aufbauphase wichtig zu erkennen, dass der Umgang mit Open Access-Publikationsgebühren keine isolierte Herausforderung darstellt, sondern im Zentrum der strategischen und organisatorischen Weiterentwicklung der Informationsversorgung von wissenschaftlichen Einrichtungen steht. So ist ein ausgewiesener Etat für Publikationsgebühren weit mehr als nur eine weitere Kostenstelle im institutionellen Gefüge. Er ist vielmehr zeitgemäßer Ausdruck der sich wandelnden Anforderungen und Erwartungen in der wissenschaftlichen Kommunikation und zudem ein zentrales Instrument, um die ökonomischen Beziehungen einer wissenschaftlichen Einrichtung zu den Verlagen neu zu organisieren.

Ein Publikationsfonds ergänzt die heute tendenziell zu eng gefasste Erwerbungspraxis an den Bibliotheken und erweitert die strategischen Gestaltungsspielräume von wissenschaftlichen Einrichtungen gegenüber den Verlagen. Es greift zu kurz, wenn (knappe) Gelder nur in dem Silo „Bibliothekserwerbungsetat" mit seiner recht eindimensionalen Logik verwaltet werden und gleichzeitig für andere, von Wissenschaftlern zunehmend gewünschte Dienstleistungen rund um die

\footnotetext{
${ }^{1}$ Zur generellen Einführung in Open Access und zur Fallunterscheidung zwischen dem grünen Weg (Parallelveröffentlichung i.d.R. in Repositorien) und dem goldenen Weg (unmittelbare Veröffentlichung nach Open Access-Kriterien) bietet sich die Informationsplattform Open Access an, siehe http://open-access.net.
} 
wissenschaftliche Kommunikation keine ausreichende Finanzierung und keine ausreichende Unterstützung vorgesehen sind.

Ausgehend von einer Beschreibung der aktuellen Open Access-Debatte und ihrer inhaltlichen Stoßrichtung sollen in diesem Beitrag die anstehenden Herausforderungen für die Ausrichtung und Organisation von Bibliotheken herausgearbeitet werden. 2 Dabei wird die These vertreten, dass wichtige bibliothekspolitische Weichen neu gestellt werden müssen und insbesondere der Erwerbungsetat und die hinter diesem stehende Praxis für den neuen Kontext vorzubereiten sind. Ohne eine solche Re-Kontextualisierung droht den Bibliotheken ein sukzessiver Bedeutungsverlust in der Informationsversorgung für die Wissenschaft, was zunächst zu einer Erosion der Aufgaben, am Ende möglicherweise gar zu einem weitgehenden Verlust der Etatmittel führen könnte.

\section{Open Access und der anschwellende Ruf nach Transformation der Subskriptionskosten}

Zentrale internationale Veranstaltungen wie die letzten beiden Folgekonferenzen zur „Berliner Erklärung" im Oktober 2010 in Peking und November 2011 in Washington zeigen, dass die Beschäftigung mit dem goldenen Weg zu Open Access erheblich an Fahrt aufgenommen hat. ${ }^{3}$ Immer deutlicher schält sich der Konsens heraus, dass das genuine Open Access-Publizieren anzustreben und entsprechend finanziell zu unterfüttern sei. Mit zunehmender Häufigkeit wird die Frage gestellt, welche Bedingungen geschaffen werden müssen, damit auch etablierte Zeitschriften von ihrem bisherigen Subskriptionsmodell auf die Finanzierung über Publikationskosten umgestellt werden können. Diese Transformation wird als die zentrale Herausforderung in der Open Access-Debatte begriffen.

Auf Basis dieser Erkenntnis arbeiten weltweit inzwischen viele Arbeitsgruppen und Gremien, Wissenschaftsorganisationen und Forschungsförderer, Verlage und Informationseinrichtungen an Konzepten und Handlungsanleitungen für die entsprechende Umsetzung. Ohne diese Aktivitäten erschöpfend darstellen zu können, sollen im Folgenden einige der wichtigsten Entwicklungen schlaglichtartig beleuchtet werden:

- Wissenschaftspolitik auf europäischer Ebene: Im Juli 2009 haben die European Heads of Research Councils (EUROHORCs) und die European Science Foundation (ESF) ihre programmatische "Vision on a Globally Competitive Era and their Road Map for Action" vorgelegt. ${ }^{4}$ In diesem Handlungsplan, der in Bezug auf Open Access zu den ambitioniertesten überhaupt gehört, heißt es: „The aim is a system of scientific publications in which free access to all (published) scientific information is guaranteed. This involves a move toward Full Open Access. Ultimately, this means replacing the present reader-paid publication system with an author- or

\footnotetext{
${ }^{2}$ Der Beitrag ist eine Ausarbeitung zweier Vorträge, die der Verfasser am 5. Oktober 2011 im Rahmen der Open Access-Tage in Regensburg und am 24. Mai 2012 auf dem 101. Deutschen Bibliothekartag in Hamburg gehalten hat.

${ }^{3}$ Die „Berliner Erklärung über den offenen Zugang zu wissenschaftlichem Wissen“ vom Oktober 2003 (http://oa.mpg.de/lang/de/berlin- prozess/berliner-erklarung/) ist nach wie vor eines der wichtigsten Bezugsdokumente der Open Access-Debatte und wurde bisher welt- weit von 385 Institutionen unterzeichnet (Stand: 31.07.2012). Zur Dokumentation der etwa jährlich stattfindenden Folgekonferenzen siehe http://oa.mpg.de/lang/de/berlin-prozess/berlin-konferenzen/.

${ }^{4}$ Vgl. http://www.eurohorcs.org/SiteCollectionDocuments/ESF_Road\%20Map_long_0907.pdf.
} 
institution-paid one." ${ }^{5}$ Die daraufhin von EUROHORCs zu Open Access eingesetzte Arbeitsgruppe wird seit 2011 auch unter dem neuen wissenschaftsorganisatorischen Dach Science Europe ${ }^{6}$ weitergeführt. Sie hat bereits mehrere Zwischenberichte und Empfehlungen zur angestrebten Transformation vorgelegt. Aber auch die Europäische Kommission ist in den letzten Jahren mit vielen Stellungnahmen und Fördermaßnahmen zu Open Access hervorgetreten. Neben der Finanzierung von maßgeblichen und breit rezipierten Open Access-Projekten wie SOAP $^{7}$ und $\mathrm{PEER}^{8}$ ist sie insbesondere auch für die wissenschaftspolitische Steuerung in Europa zuständig. Erst am 17. Juli 2012 hat die Europäische Kommission unter der Überschrift „Towards better access to scientific information" angekündigt, dass Open Access für Forschungsergebnisse als ein Grundprinzip für Horizon 2020, das neue EU-Förderprogramm für Forschung \& Innovation in der Periode 2014-2020, verankert wird. Demnach stehen alle ab 2014 mit EU-Mitteln geförderte Publikationen unter einer Open Access-Anforderung. Es werden Vorkehrungen getroffen, dass die entsprechenden Publikationskosten über die jeweilige Projektförderung abgerechnet werden können. ${ }^{9}$

- Wissenschaftspolitik auf internationaler Ebene: Auch über den europäischen Raum hinaus sind immer mehr konzertierte Aktivitäten zur Förderung von Open Access zu verzeichnen. In fast allen relevanten Wissenschaftsstandorten der Welt befassen sich die nationalen Forschungsförderer, Wissenschaftsministerien und wichtigsten Forschungseinrichtungen in der einen oder anderen Weise mit diesem Thema. Und sogar in supranationalen Gremien steht Open Access immer häufiger auf der Agenda. So wurde z.B. im neuen Global Research Council bei dessen erstem Treffen im Mai 2012 in Washington die Bedeutung von Open Access sehr deutlich herausgearbeitet. Entsprechend wird Open Access auch zu einem Schwerpunkt des nächsten Treffens im Mai 2013 in Berlin, ausgerichtet von der Deutschen Forschungsgemeinschaft (DFG), sein.10 Als ein weiterer wichtiger internationaler Schub hin zum unmittelbaren Open AccessPublizieren ist die vielbeachtete Gründung der neuen Zeitschrift eLife zu erwähnen, die gemeinsam getragen von Wellcome Trust in Großbritannien, dem Howard Hughes Medical Institute in den USA und der Max- Planck-Gesellschaft in Deutschland - neue Maßstäbe in der wissenschaftlichen Kommunikation setzen soll. ${ }^{11}$ Die Aufbauphase dieser Zeitschrift ist abgeschlossen. Derzeit werden die ersten Publikationen akquiriert, mit deren Veröffentlichungen bis Ende 2012 zu rechnen ist.

\footnotetext{
${ }^{5}$ Anm. 4, S. 17.

${ }^{6}$ Vgl. http://www.scienceeurope.org/.

${ }^{7}$ Das Projekt SOAP (Study of Open Access Publishing) untersuchte von 2009 bis 2011 die Angebote von Open Access-Zeitschriften und führte eine sehr breit angelegte Befragung von Wissenschaftlern hinsichtlich ihrer Einstellung zu Open Access durch; siehe http://project- soap.eu/.

${ }^{8}$ Das Project PEER (Publishing and the Ecology of European Research) untersuchte in einem großen Feldversuch von 2008 bis 2012, wie sich das systematische Einspeisen von Publikation in ihrer finalen Autorenversion in Repositorien ("grüner Weg" des Open Access) auf das Leserverhalten und die ökonomische Grundlage von Zeitschriften auswirkt; siehe http://www.peerproject.eu/.

${ }^{9} \mathrm{Vgl}$. http://ec.europa.eu/research/science-society/index.cfm?fuseaction=public.topic\&id=1301.

${ }^{10} \mathrm{Vgl}$. http://www.globalresearchcouncil.org/. Zu inhaltlichen Berichten über das erste Treffen im Mai 2012 siehe die News Blogs von Nature: Richard van Noorden, World's science funders announce Global Research Council (http://blogs.nature.com/news/2012/05/ worlds-science-funders-announce-global-researchcouncil.html) und Science: David Malakoff, New Global Research Council Takes Off (http://news.sciencemag.org/scienceinsider/2012/05/new-global- research-council-take.html).

${ }^{11} \mathrm{Vgl}$. http://www.elifesciences.org/.
} 
- Aktivitäten in Deutschland: Nicht nur durch die Berliner Erklärung von 2003 gehört Deutschland schon seit langem zu den besonders aktiven Ländern in der Open Access-Debatte. In der Schwerpunktinitiative "Digitale Information“ der Allianz der deutschen Wissenschaftsorganisationen stellt Open Access eines der konkreten Handlungsfelder dar. ${ }^{12}$ Die entsprechende Arbeitsgruppe tritt immer wieder mit wichtigen forschungspolitischen Beiträgen in Erscheinung. ${ }^{13}$ An jeder der außeruniversitären Forschungseinrichtungen in Deutschland sowie an zahlreichen Hochschulen ist Open Access inzwischen personell und inhaltlich verankert. Für diese Entwicklung leistet vor allem auch die DFG wertvolle Unterstützung, indem entsprechende Richtlinien verabschiedet und Förderlinien aufgesetzt werden. ${ }^{14}$ Dabei ist insbesondere das 2009 eingerichtete Programm „Open Access Publizieren“ von besonderer Relevanz. ${ }^{15}$ Hier können wissenschaftliche Hochschulen bei der DFG Mittel zum Aufbau eines Publikationsfonds einwerben, um ihre Autoren bei der Finanzierung von Artikel-Publikationsgebühren zu unterstützen. Damit können Publikationskosten für Beiträge in reinen Open-Access-Zeitschriften mit fachlich anerkannten Review-Verfahren bis zu einer Höhe von 2.000 Euro übernommen werden. Von dieser Förderung sollen gezielt Impulse für die Umschichtung bestehender Budgets hin zu einem Etat, aus dem künftig Open-Access-Publikationsgebühren finanziert werden, ausgehen. Daher müssen antragstellende Hochschulen ein Viertel des prognostizierten Mittelbedarfs aus dem eigenen Forschungs- oder Bibliotheksbudget speisen. In den Jahren 2010 und 2011 profitierten insgesamt 19 Universitäten, denen Mittel in Höhe von insgesamt 1,18 Mio. Euro bewilligt werden konnten, von dem neuen Förderinstrument. ${ }^{16}$

- Aktivitäten in Großbritannien: Open Access erfährt auch in Großbritannien seit längerer Zeit besondere Aufmerksamkeit. In den vergangenen Jahren wurde eine Vielzahl von Studien veröffentlicht, in denen einzelne Aspekte von Open Access untersucht wurden, allerdings meist mit einem auf Großbritannien eingeschränkten Fokus. Anfang Dezember 2011 hat die britische Regierung das Dokument "Innovation and Research Strategy for Growth“ veröffentlicht. ${ }^{17}$ Darin bekennt sich die britische Regierung zu einer Gewährleistung des freien und offenen Zugangs zu den Ergebnissen öffentlich geförderter Forschung. Der britische Wissenschaftsminister David Willetts betonte in diesem Zusammenhang ausdrücklich die Bedeutung von Open Access: „The Government believes that published research material which has been publicly financed should be publicly accessible - and that principle goes well beyond the academic community. ${ }^{18}$ Willetts hat im gleichen Jahr eine "Working Group on Expanding Access to Published Research Findings" („,Finch Group“) eingesetzt, in der Vertreter der Research Councils UK (RCUK), von Förderorganisationen, der Verlage, der Universitäten, Bibliotheken und weiterer Interessengruppen die Auswirkungen von Open Access auf Großbritannien analysiert und Empfehlungen für die weitere Umsetzung an die Regierung und anderen Beteiligten erarbeitet

\footnotetext{
${ }^{12}$ Vgl. http://www.allianzinitiative.de.

${ }^{13}$ Vgl. http://www.allianzinitiative.de/de/handlungsfelder/open_access/arbeitsgruppe/.

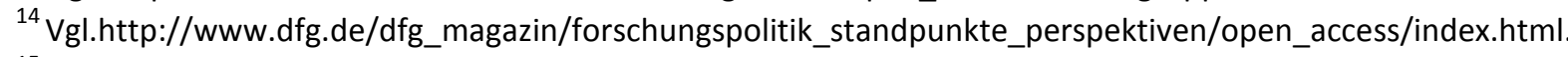

${ }^{15}$ Vgl. http://www.dfg.de/formulare/12_20/12_20.pdf.

${ }^{16}$ Angaben nach Eppelin A. et al., Zur Beschäftigung mit Publikationsfonds in Deutschland, GMS Med Bibl Inf 2012; 12(1-2). DOI: 10.3205/mbi000240.

${ }^{17} \mathrm{Vgl}$. http://www.bis.gov.uk/innovatingforgrowth

${ }^{18}$ Rede von Wissenschaftsminister Willetts auf der Jahreshauptversammlung des Britischen Verlegerverbandes, London, 2. Mai 2012; vgl. http://www.bis.gov.uk/news/speeches/david-willetts-public-access-to-research.
} 
haben. In dem Bericht („Finch Report“), veröffentlicht im Juni 2012, ${ }^{19}$ wird ein klares Bekenntnis zum Übergang zu Open Access abgelegt, und es werden klare politische Weichenstellungen und eine entsprechende Finanzierung, um den Umstieg auf ein Publikationskostenmodell herbeizuführen, gefordert. In Reaktion auf den Finch Report haben die Research Councils UK am 16. Juli 2012 eine neue Open Access Policy veröffentlicht, ${ }^{20}$ die ab April 2013 in Kraft treten wird. Darin werden mehrere konkrete Maßnahmen des grünen und goldenen Weges zu Open Access festgelegt, ${ }^{21}$ insbesondere aber eine Erklärung zur Übernahme von Publikationskosten abgegeben. Dieser Vorstoß von RCUK erfolgte in enger Abstimmung mit der britischen Regierung, die am gleichen Tag die Ergebnisse des Finch Reports offiziell begrüßte und sich ausdrücklich bis auf den Aspekt der Mehrwertsteuer alle Empfehlungen zu eigen machte: „We wish to extend open access to research and so accept all the conclusions in the report." ${ }^{22}$ Zusätzlich wurden weitere Maßnahmen seitens der Regierung und der öffentlichen Forschungsförderer in England angekündigt. ${ }^{23}$ Aber auch die private Stiftung Wellcome Trust, ein wichtiger Förderer der Grundlagenforschung, gab Ende Juni 2012 bekannt, dass ihre seit 2006 bestehende Open AccessPolicy mit sofortiger Wirkung dahingehend verschärft wird, dass die Einhaltung der Auflage des Open-Access-Publizierens stärker überwacht und mit Sanktionen bewehrt wird. ${ }^{24}$

- Aktivitäten der Verlage: Auch auf Seiten der Verlage ist spätestens seit zwei bis drei Jahren ein deutliches Umdenken zu erkennen. Ein interessanter Gradmesser für diesen Umschwung ist sicherlich die seit 2006 jährlich in Berlin stattfindende internationale Tagung „Academic Publishing in Europe", an der neben vielen Verlagsvertretern auch Repräsentanten aus der Politik, der Wissenschaft und von Serviceeinrichtungen des Informationswesens teilnehmen. Wurden hier in den ersten Jahren noch deutliche Vorbehalte gegen Open Access artikuliert, so werden inzwischen Beiträge wie der im Januar 2012 von Bernard Schutz, Direktor am Max-Planck-Institut für Gravitationsphysik in Golm, über „Enabling the Transition of Existing Journals to Open Access" mit großem Interesse und starkem Zuspruch aufgenommen. ${ }^{25}$ Vor allem bei den international agierenden naturwissenschaftlichen Verlagen hat Open Access längst seinen Schrecken verloren und wird inzwischen sogar als relevantes Geschäftsmodell der Zukunft propagiert. So befasst sich beispielsweise die International Association of Scientific, Technical \& Medical Publishers (STM) als Dachverband auf ihren Tagungen immer häufiger mit den Aspekten von Open Access (z.B. auch 2012 wieder mit einer Veranstaltung im Vorfeld der Frankfurter Buchmesse). Nachdem STM 2007 noch die eher gegen Open Access gerichtete „Brüsseler Erklärung“ organisiert hatte, ${ }^{26}$ trat der Verband Anfang 2012 mit der klar den goldenen Weg des Open Access befürwortenden Erklärung „Publishers Support Sustainable Open Access“ in Erscheinung. ${ }^{27}$ Im Sommer 2012 haben bereits gut 50 Verlage diese Deklaration unterzeichnet - und damit innerhalb sehr kurzer Zeit nur

\footnotetext{
${ }^{19} \mathrm{Vgl}$. http://www.researchinfonet.org/wp-content/uploads/2012/06/Finch-Group-report-FINAL-VERSION.pdf.

${ }^{20} \mathrm{Vgl}$. http://www.rcuk.ac.uk/documents/documents/RCUK\%20_Policy_on_Access_to_Research_Outputs.pdf.

${ }^{21}$ Vgl. dazu Anm. 1

${ }^{22}$ Vgl. http://www.bis.gov.uk/assets/biscore/science/docs/l/12-975-letter-government-response-to-finchreport-research-publications.pdf.

${ }^{23}$ Anders als in Großbritannien gibt es in Deutschland bisher keine offizielle Stellungnahme zu Open Access von der Bundesregierung.

${ }^{24}$ Vgl. http://www.wellcome.ac.uk/News/Media-office/Press-releases/2012/WTVM055745.htm.

${ }^{25} \mathrm{Vgl}$. Video des Vortrags unter: http://river-valley.tv/enabling-the-transition-of-existing-journals-to-openaccess/.

${ }^{26} \mathrm{Vgl}$. http://www.stm-assoc.org/brussels-declaration/.

${ }^{27} \mathrm{Vgl}$. http://www.stm-assoc.org/publishers-support-sustainable-open-access.
} 
geringfügig weniger als noch vor fünf Jahren die Brüsseler Anti-Erklärung.

- SCOAP3 als wichtiges Präzedenztransformationsprojekt: Eine Übersicht über die internationalen Transformationsaktivitäten im Zeitschriftenbereich bleibt unvollständig, geht man nicht auf das „Sponsoring Consortium for Open Access Publishing in Particle Physics“ (SCOAP3) ein, ${ }^{28}$ die „Mutter" aller diesbezüglichen Vorhaben. Konzeptionell und organisatorisch am CERN beheimatet, soll mit Hilfe eines weltweit aktiven Konsortiums die Umstellung der für die Hochenergieforschung wichtigsten Zeitschriften auf ein Open Access-Modell ermöglicht werden. Die bisherigen Subskriptionsgebühren für diese Zeitschriften sollen dabei entfallen und über das Konsortium in einen Fonds umgeleitet werden, aus dem dann die Publikationsdienstleistungen der Verlage - die Organisation der Qualitätssicherung durch das Peer Review-Verfahren und Bereitstellung der Publikationen- direkt bezahlt werden. Nach einigen Jahren der Vorbereitung tritt SCOAP3 nun in die entscheidende Phase ein: Über ein Ausschreibungsverfahren konnten die zwölf an diesem Programm teilnehmen Zeitschriften von insgesamt sieben Verlagen ermittelt und bekannt gegeben werden. ${ }^{29}$ Zwischen Oktober 2012 und Frühjahr 2013 werden zum einen die konkreten Beitrittsvereinbarungen zu SCOAP3 geschlossen und zum anderen die Entflechtung und Umleitung der bisherigen Subskriptionskosten für diese zwölf Zeitschriften in Zusammenarbeit mit den Verlagen organisiert. Aus Deutschland beteiligen sich die Max-PlanckGesellschaft und die Helmholtz-Gemeinschaft, repräsentiert durch das Deutsche ElektronenSynchrotron (DESY), an SCOAP3. Mit Unterstützung durch die DFG koordiniert die Technische Informationsbibliothek in Hannover die Beteiligung der Hochschulen und weiterer Einrichtungen in Deutschland. ${ }^{30}$ Die Vorbereitungen sollen 2013 abgeschlossen werden, so dass die Transformationen ab dem Publikationsjahrgang 2014 wirksam werden und das SCOAP3Konsortium dann seinen Regelbetrieb aufnimmt.

\section{Auswirkung auf Ausrichtung und Organisation von Bibliotheken und ihrer Etatpolitik}

Ein großer Teil der Beschleunigung in der Open Access- Diskussion geht auf entsprechende Positionierungen von Forschungsförderern, außeruniversitären Forschungseinrichtungen, Forschungsministerien und Wissenschaftsverbänden zurück - sowie zunehmend auch auf die Neuausrichtung von Verlagen. Doch an den Hochschulen und vor allem auch an den Hochschulbibliotheken gehen diese Entwicklungen zum Teil noch recht deutlich vorbei - nicht nur in Deutschland. Der Alltag in der Mehrzahl der Bibliotheken nimmt weiter seinen gewohnten Verlauf, obwohl bereits die Anzeichen deutlicher Umbrüche zu erkennen sind, die vor den Bibliotheken keineswegs Halt machen werden. Gerade in Anbetracht der heraufziehenden Herausforderungen wäre es aber wichtig, sich gegenüber den absehbaren Umbrüchen und Transformationen nicht nur nachgelagert und passiv zu verhalten, sondern sich durch vorausschauendes Handeln eigene Gestaltungsspielräume zu erarbeiten. Insgesamt gilt es, das Bewusstsein dafür noch weiter zu schärfen, dass den Universitäten und anderen wissenschaftlichen Einrichtungen eine wichtige Verantwortung für die Ausgestaltung der für den Wandel notwendigen Strukturen zukommt und sie nicht auf die Umstellung der Geschäftsmodelle durch die Verlage abwartend verharren können. Der Umstieg auf Open Access im Sinne des goldenen Weges ist nur durch proaktives Zutun seitens der

\footnotetext{
${ }^{28}$ Vgl. http://www.scoap3.org/.

${ }^{29}$ Vgl. http://www.scoap3.org/news/news94.html.

${ }^{30}$ Siehe dazu die eigenen deutschen Projektseiten zu SCOAP ${ }^{3}$ unter http://www.scoap3.de/.
} 
Wissenschaft effektiv und nachhaltig zu erreichen.

In Sinne dieser Gedanken möchte ich die anstehenden Herausforderungen in drei aufeinander aufbauenden Überlegungen beschreiben und dabei insbesondere auch die organisatorischen und strategischen Implikationen für den Bibliotheksalltag herausarbeiten:

\subsection{Die Einrichtung von Publikationsfonds ist ein Gebot der Stunde und ermöglicht den Einstieg in die notwendigen neuen Prozesse und Strukturen.}

Seit den Tagen der Berliner Erklärung, also seit inzwischen fast 10 Jahren, wird an Open AccessUmsetzungen entweder nach dem grünen oder goldenen Weg gearbeitet. ${ }^{31}$ Konzentrierte sich die Debatte in den ersten Jahren noch stärker auf den grünen Weg, so haben sich die Gewichte in den letzten Jahren auf den goldenen Weg verlagert. Insbesondere die außeruniversitären Forschungseinrichtungen in Deutschland, allesamt Erstunterzeichner der Berliner Erklärung, verfügen inzwischen - zum Teil bereits seit mehreren Jahren - über Publikationsfonds mit entsprechenden internen Regelungen und Erfahrungen. In der Max-Planck-Gesellschaft beispielsweise wurde mit der zentralen Übernahme von Publikationskosten im Jahr 2003 begonnen. Auf der Basis von entsprechenden Vereinbarungen mit Open Access-Verlagen, die zahlenmäßig und inhaltlich immer weiter ausgebaut wurden, konnten im Jahr 2011 bei 476 Zeitschriftenveröffentlichungen aus den Max-Planck-Instituten die Publikationskosten von durchschnittlich etwa 925 Euro zentral übernommen werden. Bereits 2005 wurde in der Max-Planck-Gesellschaft die wichtige strategische Entscheidung getroffen, die Subskriptions- und Publikationskosten aus ein und demselben Etat zu bestreiten, um so maximale Steuerungsfähigkeit unter einheitlicher Organisationsstruktur und Prozessverantwortung zu gewährleisten. ${ }^{32}$ Auch die anderen außeruniversitären Forschungseinrichtungen in Deutschland haben in den letzten Jahren ähnliche Prozesse durchlaufen bzw. Regelungen geschaffen und bekennen sich zur Publikationskostenübernahme für ihre Autoren. ${ }^{33}$

Erfreulicherweise setzen sich inzwischen auch immer mehr Universitäten in Deutschland mit den Anforderungen der Publikationskostenübernahme für die Hochschulangehörigen auseinander. Hilfreich ist dabei seit 2009 die oben beschriebene Förderlinie der DFG zum gezielten Aufbau von Publikationsfonds in Deutschland. Dass die Wissenschaftseinrichtungen in Deutschland mit ihren Maßnahmen insgesamt auf einem richtigen Weg liegen, zeigen die Ergebnisse der SOAP-Studie: Zum einen ist die Zustimmung zu Open Access unter deutschen Wissenschaftlern mit 89,7 Prozent beachtlich hoch; zum anderen wird ein besserer Zugang zu Open Access-Fördermitteln angegeben als in allen anderen Ländern - wobei aber auch der Wunsch nach Verbesserungen weiterhin zu Protokoll gegeben wird. $^{34}$

\footnotetext{
${ }^{31}$ Vgl. dazu Anm. 1

${ }^{32}$ Siehe dazu http://www.biomedcentral.com/funding/maxplancksociety.

${ }^{33}$ Siehe dazu: Pampel H., Liebenau L. Umgang mit Open-Access-Publikationsgebühren - Praxis und Perspektive in der Helmholtz-Gemeinschaft. Bibliothek Forschung und Praxis. 2012;36(1):110-6. DOI: 10.1515/bfp-20120013 sowie Eppelin A. et al. (Anm. 16).

${ }^{34}$ Angaben nach Dallmeier-Tiessen S., Lengenfelder A. Open Access in der deutschen Wissenschaft - Ergebnisse des EU-Projekts „Study of Open Access Publishing“ (SOAP). GMS Med Bibl Inf. 2011;11(1-2). Doc03. DOI: 10.3205/mbi000218; zur Dokumentation des SOAP-Projekts siehe auch Anm. 7
} 
Mit der Einrichtung von Publikationsfonds sind Anforderungen an die entsprechenden Prozesse und Fragen nach der generellen Steuerungsfähigkeit eng verbunden. Die Aufbauphase sollte insbesondere auch dazu genutzt werden, wichtige Finanzkenngrößen, die bis dato zwar anfallen, aber in der Regel nicht zentral erfasst werden, systematisch zu erheben. Dazu gehören im Wesentlichen alle Kosten für Verlagsdienstleistungen, z.B. „herkömmliche“ Publikationsgebühren wie „page charges“, Einreichgebühren oder Abbildungskosten. Gerade unter Nachhaltigkeitsaspekten und für die strategische Weiterentwicklung des Publikationsfonds ist eine möglichst ganzheitliche Erfassung des Mittelabflusses an die Verlage von großer Wichtigkeit. Ebenso ist die bessere Erhebung der Publikationskennzahlen der eigenen Einrichtung (Anzahl der Publikationen insgesamt und Verteilung auf Verlage, Anteil Kollaborationen und Co-Autoren etc.) fast schon eine Voraussetzung für die Organisation und Steuerung eines Publikationskostenprogramms. Neben den großen kommerziellen bibliographischen Datenbanken ist für derartige Aufstellungen insbesondere auch das eigene Open Access-Repositorium ein guter Ausgangspunkt.

Für den Start eines Publikationskostenprogramms sind wichtige Festlegungen zu treffen: Nach welchen (Qualitäts-) Kriterien einer Zeitschrift und bis zu welcher Höhe sollen Kosten gefördert werden? Sollen Mindeststandards für die Einräumung von Nutzungsrechten definiert werden? Sollen die Kosten pro Kopf oder pro Fachbereich gedeckelt werden? Sind dezentrale Kostenbeteiligungen vorgesehen? Sind Drittmittel wo möglich vor Eigenmitteln einzusetzen? Sollen die Rechnungen zentral einlaufen oder von den Autoren an eine zu definierende Stelle weitergleitet werden? Wie soll die weitere Rechnungsbearbeitung organisiert werden? Welche Maßnahmen zur Bekanntmachung und Werbung in der eigenen Einrichtung sind zu ergreifen? Welche Mechanismen und Intervalle zur Evaluation des Programms sind vorgesehen? Und wer ist überhaupt wofür verantwortlich?

\subsection{Ein Publikationskostenfonds ist nicht nur eine neue Kostenstelle, sondern ein zentrales Instrument, um die ökonomischen Beziehungen zu den Verlagen neu zu organisieren. Die Bibliotheken sind dazu prädestiniert, müssen sich aber entsprechend weiterentwickeln.}

Die erste Überlegung hat uns die Bedeutung der Einrichtung eines Publikationsfonds und dessen alltagspraktischen Anforderungen vor Augen geführt. In der zweiten Überlegung geht es darum zu erkennen, dass sich die Beschäftigung mit einem Publikationsfonds nicht in den Prozessanforderungen erschöpfen darf, sondern unweigerlich in einen größeren operativen und strategischen Zusammenhang einzubetten ist. In diesem Abschnitt wird nun also der Übergang von den organisatorischen Fragen zu den strategischen Fragen beschritten.

Es gehört zum Selbstverständnis der Bibliotheken, für die Informationsversorgung ihrer Einrichtung zuständig zu sein. Doch durch die technischen Möglichkeiten - nicht nur durch die Digitalisierung selbst, sondern auch durch die zunehmend webbasierte wissenschaftliche Kommunikation - verändert sich das Verständnis und die Möglichkeit dessen, was Informationsversorgung heute ausmacht. Dabei wird immer deutlicher, dass ein Verständnis von Informationsversorgung, das sich auf die Ermöglichung des lesenden Zugriffs beschränkt, zu kurz greift und wesentliche Bedürfnisse seitens der Wissenschaftler unberücksichtigt lässt. Wichtige Anforderungen aus der Wissenschaft, z.B. nach Nachnutzung von Inhalten in anderen Kontexten, nach Verwirklichung von einrichtungsüberreifender Kollaboration in Forschungs- und Publikationsprojekten, nach maschinenlesbarer Nutzung von Inhalten oder nach einem 
systematischen Aufbau von fachspezifischen Volltextsuchmöglichkeiten, finden in der gegenwärtigen Lizenzierungspraxis noch keine hinreichende Berücksichtigung. Deshalb ist es wichtig, dass die Bibliotheken ihre Perspektive erweitern und erkennen, dass sie nicht nur die erforderlichen Rechte zum Lesen der Inhalte für ihre wissenschaftlichen Nutzer zu beschaffen haben, sondern an sich dazu prädestiniert sind, die Interessen ihrer Einrichtung in ihrer maximalen Gesamtheit zu bündeln und in den Austausch- und Verhandlungsprozessen mit den Verlagen so wirksam wie möglich einzubringen. Deshalb ist ein Publikationsfonds nicht nur eine neue Kostenstelle irgendwo im System, sondern ein strategisches Instrument, um die ökonomischen Beziehungen der eigenen Einrichtung gegenüber den Verlagen neu zu organisieren und dabei die institutionellen Interessen zeitgemäß, umfassend und auf Augenhöhe mit den Verlagen zum Ausdruck zu bringen.

Die Open Access-Debatte der letzten 10 Jahre ist leicht zu dechiffrieren als ein Ausdruck sich wandelnder Möglichkeiten und Anforderungen in der wissenschaftlichen Kommunikation. Auch die Erwerbungspraxis an den Bibliotheken muss dies erkennen und sich konzeptionell und organisatorisch entsprechend erweitern. Gewisse Entwicklungen in diese Richtung liegen bereits vor. So versuchen immer mehr Bibliotheken und Konsortien, mit den ausgehandelten Leserechten auch technische Aspekte, ausdrückliche Archiv- und Hostingrechte oder seit neuem auch Open Access-Optionen zu verbinden. Von besonderem Gewicht sind dabei die Standards, die mit Einführung der Allianz-Lizenzen in Deutschland seit dem Lizenzjahr 2011 verbunden und in den „Grundsätzen für den Erwerb DFG-geförderter überregionaler Lizenzen“ niedergelegt sind. ${ }^{35}$ Auch eine Open Access-Komponente-das Recht zur Speicherung von Publikationen aus den teilnehmenden Einrichtungen in einem Online- Repositorium - konnte dadurch in den AllianzLizenzen verankert werden. ${ }^{36}$ Dies ist ein Meilenstein auf dem Weg, Open Access-Anforderungen und die herkömmliche Lizenzierungspraxis der Bibliotheken und Konsortien in einen gemeinsamen und einheitlichen Kontext zu bündeln. In dieser Hinsicht sind auch weitere Überlegungen und Anstrengungen seitens der Allianz-Initiative "Digitale Information“ in der zweiten Durchführungsperiode 2013-2017 vorgesehen.

Für die weitere Entwicklung der Bibliotheken wird auch noch die Frage nach ihrer Rolle und Zuständigkeit sehr wichtig sein. Damit ist hier weniger die personelle Zuständigkeit im operativen Sinne gemeint, sondern die Verankerung der generellen Verantwortung für die Bündelung und das Aushandeln der Wissenschaftskommunikationsinteressen gegenüber den Verlagen. Es geht hier also um elementare Fragen der Steuerung wissenschaftspolitischer Interessen. Eine derartige Zuständigkeit könnte beispielsweise im Präsidialbereich einer wissenschaftlichen Einrichtung angesiedelt oder aber auch der Bibliothek übertragen werden, die bereits über Personal, Kompetenzen, Verlagskontakte und einen großen Teil des einzusetzenden Etats verfügt. Wegen dieser an sich vorhandenen Vorteile spricht im Moment alles noch dafür, dass die Bibliothek die

\footnotetext{
${ }^{35}$ Verabschiedet im Juni 2010; vgl.

http://www.dfg.de/download/programme/wissenschaftliche_literaturversorgung_informationssysteme/antrag stellung/12_18/12_18.pdf

${ }^{36}$ Siehe dazu die beiden Beiträge in diesem Heft: Hillenkötter, C.: Die Open Access-Komponente in den DFGgeförderten Allianz-Lizenzen. In diesem Heft S. 300-304; Stöber A., Open-Access-Rechte in Alli- anz- und Nationallizenzen. Eine Handreichung für Repository-Mana- ger, Bibliothekare und Autoren. In diesem Heft S. 364-368. Beitrag auch unter http://www.allianzinitiative.de/fileadmin/handreichung_allianz_lizenzen.pdf. DOI: 10.2312/allianzoa.004
} 
erweiterten Funktionen auch in Zukunft übernimmt. Doch dazu müssen sich die Bibliotheken noch stärker für den Wandel in der wissenschaftlichen Kommunikation öffnen und neu über ihren Auftrag und ihr Dienstleistungsspektrum nachdenken. Es ist gewiss eine der großen aktuellen Herausforderungen für die Bibliotheken, die sich ändernden Anforderungen an die Informationsversorgung wahrzunehmen und die eigene Ausrichtung entsprechend weiter zu entwickeln. Wird die zentrale strategische Verantwortung erst einmal an anderer Stelle etabliert und werden dort Personal sowie Kompetenzen aufgebaut, dann wird es für die Bibliotheken sehr schwer, einmal verlorenes Terrain zurückzugewinnen. In einen solchen Falle wäre wohl sogar eher damit zu rechnen, dass nach verlorener Zuständigkeit am Ende der Bibliothek auch noch wichtige Etatmittel verloren gingen, weil in naher Zukunft nicht mehr der lesende Zugriff auf die Informationsinhalte der entscheidende Kostenfaktor sein wird, sondern die Gebühren für die Veröffentlichung und möglichweise für weitere neue Dienstleistungen jenseits des bisherigen Bibliotheksspektrums.

\subsection{Die Übernahme von Publikationskosten kann nicht maximal ausgedehnt werden, ohne die Subskriptionskosten zu transformieren. Der Erwerbungsetat der Bibliotheken wird zum konstitutiven Teil der Open Access-Transformation.}

Damit sind wir in unseren Überlegungen bei der Erfassung und Organisation der Finanzströme angelangt. Meistens wird heute noch so getan, als habe das eine-der für die herkömmliche Informationsversorgung vorgesehene Erwerbungsetat - mit dem anderen - dem auf- und auszubauenden Publikationsfonds - nichts zu tun. Doch die Realität könnte gegenteiliger nicht sein.

Steigende Zahlen bei Open Access-Publikationen und entsprechend wachsende Kosten sind in erster Linie als Ausdruck eines sich wandelnden Anspruchs der Wissenschaftler und als Ansporn für den weiteren Umbau der Finanzströme für wissenschaftliche Information zu begreifen. Denkt man den Ansatz eines Publikationsfonds zu Ende, so geht es konsequenterweise um die Transformation bestehender Zeitschriften vom bisherigen Subskriptionsmodell zu einem generellen Publikationskostenansatz. Je mehr der Ansatz ausgedehnt wird, desto mehr sind es die gleichen Zeitschriften, die entweder von der einen oder von der anderen Seite her mit Kosten zu bedienen sind. Von daher ist bei einem Publikationskostenansatz immer auch der Erwerbungsetat mit zu berücksichtigen. Die Übernahme von Publikations- kosten kann nicht maximal ausgedehnt werden, wenn nicht gleichzeitig die Subskriptionskosten entfallen - oder eben systematisch transformiert werden. Der bisherige Erwerbungsetat der Bibliotheken wird also das entscheidende fiskalische Transformationsreservoir sein.

Die Bibliotheken haben auch bei der Umgestaltung der Finanzströme die besten Voraussetzungen, durch die Einnahme einer Makro-Perspektive sowie durch die strategischorganisatorische Weiterentwicklung und inhaltliche Öffnung des Erwerbungsetats ihren Verantwortungsbereich bestätigt zu bekommen. Aber dazu müssen sie sich auch in dieser Frage weiterentwickeln und erkennen, dass der Erwerbungsetat aus heutiger Sicht zu wichtig und zu mächtig ist, um nur für den lesenden Zugriff eingesetzt zu werden. Die Bibliotheken werden die Bestimmung ihres Erwerbungsetats erweitern müssen, um ihn auch in Zukunft in der gewohnten Dimension behalten zu können. Die zunehmende Transformation zu Open Access zwingt die Bibliotheken also zu einer Re-Kontextualisierung ihres Erwerbungsetats. 


\section{Schlussbetrachtung}

Auf dem goldenen Weg zu Open Access wurden Entwicklungen in Gang gesetzt, die allem Anschein nach noch mächtige Transformationen mit sich bringen werden. Sie sind eine Reaktion auf die sich wandelnden Anforderungen in der wissenschaftlichen Kommunikation und beinhalten die strategische Reorganisation der ökonomischen Beziehungen zwischen Wissenschaftseinrichtungen und Verlagen. Dadurch wird auch ein neuer Kontext für die Bibliotheken etabliert, die ihr Selbstverständnis und ihre Praxis weiter entwickeln müssen, wenn sie ihre Zuständigkeit und ihren bisherigen Etat für die Informationsversorgung behalten wollen. Getreu dem Motto, dass nur was sich ändert, bestehen bleibt, müssen die Bibliotheken einen Teil ihrer Rolle wandeln, um ihre Rolle zu erhalten. Die Etablierung von Publikationsfonds in Verbindung mit rekontextualisierten Bibliothekserwerbungsetats ist ein wichtiger Beitrag zur Gestaltung der Informationsinfrastrukturen für eine zunehmend webbasierte Wissenschaft. 